$\xi=1$ -

\title{
A Study on Connected Domination Transition Number of a Graph
}

\author{
Kaspar $\mathbf{S}^{1}$, B. Gayathri ${ }^{2}$, D. Kalpanapriya ${ }^{1^{*}}$, M.P. Kulandaivel ${ }^{3}$ \\ ${ }^{1}$ Department of Mathematics, School of Advanced Sciences, Vellore Institute of Technology, Vellore, India - 632014. \\ ${ }^{2}$ Department of Mathematics, Periyar E.V.R. College, Tiruchirappalli, India - 620023. \\ ${ }^{3}$ Department of Information Technologies, Al Mussana College of Technology, Sultanate of Oman. \\ *Corresponding author E-mail: dkalpanapriya@vit.ac.in
}

\begin{abstract}
Since its inception, the notion of domination has found vital roles in several real life applications related to facility locations, representatives' selection, communication networks, electrical networks, etc. The vast application of the notion has paved the way for the development of the notion with several types. The notion of connected domination is a significant domination parameter amongst the several domination varieties emerged in this domain. The problem of determining limited bus stops in a route was effectively addressed by the connected domination parameter. Most of the biological and neural networks effectively use this notion to solve several problems which require the connectedness of the structures. In view of the growing applications of the variant, several researchers and scholars have published numerous research articles on the said parameter. Recently, some researchers attempted on transition of the domination parameter into a connected one. In order to facilitate this transition, another variant viz., connected domination transition number was introduced and its properties and bounds were studied. In this article we explore more properties and bounds of the parameter connected domination transition number for special types of graphs. We also characterize the instances at which the domination and connected domination parameters would be same for few types of graphs. We also attempted to derive few Nordhaus-Gaddum (NG) type results for the same.
\end{abstract}

Keywords: connected domination, domination, connected domination transition number, graph, independence number of a graph, tree.

\section{Introduction}

Though the notion of domination exists in the literature very long ago it was formalized only during the last century by Berge [1] and Ore [2]. Hundreds of research articles emerged out from then onwards about the domination parameter. The connected domination parameter was jointly introduced by Sampathkumar and Walikar in [3]. It was further studied in numerous articles such as [4], [5]. Several researchers and scholars have published numerous research articles on this domination parameter.

The backbone of any network requires to be connected. So the connected domination parameter serves as a virtual backbone in any network. In view of connectedness, the connected domination parameter has vital applications in several areas. A connected dominating set in a communication network increases the bandwidth efficiency, reduces the communication overhead, and minimizes the energy consumption. The wireless sensor networks build their virtual backbones as connected dominating sets for the effective transmission and broadcasting of network signals. The task of routing and broadcasting can be efficiently performed in ad hoc networks with the help of connected dominating sets. In view of its vital applications, Kaspar et. al have attempted to transmit a domination parameter into connected one by introducing another variant viz., connected domination transition number in [6]. The connected domination transition number for any graph $G$ was defined to be the difference between the connected domination and the domination numbers of $G$ and is denoted as $\tau_{c}(G)$. In other words, $\tau_{c}(G)=\gamma_{c}(G)-\gamma(G)$. The authors have also inves- tigated and studied the parameter for bounds and equalities for special types of graphs. In this article we focus on the connected domination transition number $\tau_{c}(G)$ and explore its few more properties in various aspects.

In any graph $G$, a subset $D$ of vertices is said to be a dominating set of $G$ if every vertex of $G$ is either a member of $D$ or adjacent to some member of $D$. A minimum dominating set is a dominating set of smallest size. The cardinality of such a minimum dominating set is referred to as the domination number of $G$ denoted by $\gamma_{c}(G)$. A dominating set $D$ of a graph $G$ is said to be a connected dominating set if the subgraph induced by the vertices of $D$ is connected. The minimum cardinality of a connected dominating set is known as the connected domination number denoted by $\gamma_{c}(G)$.

We define the connected domination transition number of a graph $G$ as the difference between the connected domination and the domination numbers of $G$ and is denoted as $\tau_{c}(G)$. In other words $\tau_{c}(G)=\gamma_{c}(G)-\gamma(G)$. We study the bounds for $\tau_{c}(G)$ and its exact values some particular classes of graphs. Further we analyze some properties on this number. In order to obtain results on the parameter $\tau_{c}(G)$, we have recalled some standard results from the literature on the domination and connected domination numbers of a graph since, this parameter depends on these two. We have made an attempt to characterize the class of graphs $G$ for which

$\gamma(G)=\gamma_{c}(G)$. Since connected domination is more stable and reliable than other such types of its kind we study the extension of a domination network into a connected domination network. 
Readers can refer to [6] to know more about the connected domination numbers transition number.

All the graphs treated in this chapter unless and otherwise specified are finite connected and nontrivial.

\section{Connected Domination Transition Number}

\section{Definition 2.1 [6]}

Let $G$ be a connected graph. The connected domination transition number is defined as the difference between the connected domination number $\gamma_{c}$ and the domination number $\gamma$ of the graph $\mathrm{G}$ and is denoted by $\tau_{c}(G)$. Symbolically, $\tau_{c}(G)=\gamma_{c}(G)-\gamma(G)$.

Since a connected dominating set exists only for connected graphs, this invariant is also defined only for connected graphs.

\section{Remark 2.2}

1. Clearly, every $\gamma_{c}$-set of a connected graph $G$ contains one or more $\gamma$-sets of $G$. Hence the connected domination transition number $\tau_{c}(G)$ can be thought of as the minimum number of vertices required to connect the vertices of any $\gamma$-set together.

2. It is same as the minimum number of intermediate vertices included in the shortest paths between the components of the subgraph induced by the vertices of some $\gamma$-set of $G$ which minimally connect them.

\section{Remark 2.3}

From the definition of the parameter $\tau_{c}$, it is evident that $\tau_{c}$ attains its maximum (minimum) value only when the parameter $\gamma_{c}$ attains its maximum value while the parameter $\gamma$ attains its mini mum value. The other extremes of these parameters yield the minimum value for the parameter $\tau_{c}$.

\section{Survey}

Before proceeding with the results on the connected domination transition number $\tau_{c}$ we recall some standard results already available in the literature survey on domination and connected domination numbers which we will use in our findings. The following result though trivial will be used in our findings.

\section{Theorem 3.1}

If $D$ is a $\gamma$-set of a connected graph $G$ with $\gamma(G) \geq 2$, then for every vertex $u \in D$, there exists at least one vertex $v \in D$ such that $d(u, v) \leq 3$.

This is a direct consequence of the fact that $D$ is a dominating set of $G$. Otherwise, there will be some vertex in the $u v$-path that is not adjacent to any vertex in $D$ implying a contradiction to the nature of the set $D$.

\section{Theorem 3.2 [3]}

For any tree $T$ of order $n, \gamma_{c}(T)=n-l$, where $l$ is the number of pendant vertices (leaves) of $T$.

Hedetniemi and R.C. Laskar derived the exact value of the connected domination number for all connected graphs in terms of the pendant vertices in some spanning tree of $G$ as below.

\section{Theorem 3.3 [4]}

If $G$ is a connected graph with $n \geq 3$, then $\gamma_{c}(G)=n-\varepsilon_{T}(G) \leq n-2$ where $\varepsilon_{T}(G)$ denotes the maximum number of pendant vertices (edges) in any spanning tree of $G$.
In [5], Arumugam and Joseph characterized the class of trees, unicyclic graphs and cubic graphs with $\gamma=\gamma_{c}$. We list out some of their results here.

\section{Theorem 3.4 [5]}

For a tree $T$ of order $p \geq 3, \gamma=\gamma_{c}$ if and only if every internal vertex is a support.

\section{Theorem 3.5 [5]}

Let $G$ be a unicyclic graph with cycle $C=u_{1} u_{2} \ldots u_{n} u_{1}, n \geq 5$ and let $X$ be the set of all vertices of degree 2 in $C$. Then $\gamma=\gamma_{c}$ if and only if the following conditions hold:

(a) Every vertex of degree at least 2 in $V-N[X]$ is a support.

(b) $\langle X\rangle$ is connected and $|X| \leq 3$

(c) If $\langle X\rangle=P_{1}$ or $P_{3}$, both vertices in $N(X)$ of degree greater than 2 are supports and if $\langle X\rangle=P_{2}$, at least one vertex in $N(X)$ of degree greater than 2 is a support.

The author also provides examples of following unicyclic graphs with $\gamma=\gamma_{c}$ in which $\langle X\rangle=P_{1}, P_{2}$ or $P_{3}$ respectively in figure 1 .

\section{Theorem 3.6 [5]}

Let $G$ be a cubic graph of order $n$. Then $\gamma=\gamma_{c}$ if and only if $G$ is isomorphic to $K_{4}, \bar{C}_{6}, K_{3,3}, G_{1}$ or $G_{2}$ where $G_{1}$ and $G_{2}$ are as given below in figure 2 .

The following upper bound on the domination number of a tree was proved by Lemanska.

\section{Theorem 3.7 [9]}

If $T$ is a tree on 3 or more vertices with $l$ pendant vertices (leaves) then $\gamma \geq \frac{n-l+2}{3}$. Also the bound is attained if and only if $T$ belongs to the family $\mathrm{R}$ where $\mathrm{R}$ is the family of all trees in which for any two distinct pendant vertices $u$ and $v, d(u, v) \equiv 2(\bmod 3)$.

The classical paper [10] of Nordhaus and Gaddum discusses about the inequalities related to the chromatic number of a graph $G$ and its complement $\bar{G}$. Among the other types of inequalities available in literature, the Nordhaus-Gaddum (NG) type inequalities are considered to be peculiar since they view a single parameter in two different ways one with the given graph $G$ itself and the other with the complement of the given graph $\bar{G}$.

\section{Theorem 3.8 [11]}

For any graph $G$, if $\gamma$ and $\bar{\gamma}$ denote the domination numbers of $G$ and $\bar{G}$ respectively, then $\gamma+\bar{\gamma} \leq n+1, \gamma \cdot \bar{\gamma} \leq n$.

Domination related NG results were first published in 1972 by Jaeger and Payan [11]. The upper bound for the sum was sharpened by Cokayne and Hedetniemi [12]. They added that $\gamma+\bar{\gamma}=n+1$, if and only if $G=K_{n}$ or $G=\bar{K}_{n}$

Analogous NG results were also developed for the connected domination number of a graph. Since the connected domination exists only for connected graphs, one can easily perceive that NG results for the parameter $\gamma_{c}(G)$ can be developed only when both the graph $G$ and its complement $\bar{G}$ are connected. The following NG result was proved by Sampathkumar and Walikar.

\section{Theorem 3.9 [3]}

If $G$ is a coconnected graph, then $\gamma_{c}+\bar{\gamma}_{c} \leq n(n-3)$, with equality if and only if $G=P_{4}$.

This result was further improved by Hedetniemi and Laskar in [4] 


\section{Theorem 3.10 [4]}

If $G$ and $\bar{G}$ are both connected, then $\gamma+\bar{\gamma} \leq n+1$.

This bound was further sharpened by Laskar and Peters [7]. They proved that the upper bound is attained if and only if $G=C_{5}$.

Many such interesting NG results on few domination parameters are discussed by Frank Harary and Teresa W. Haynes in [8].
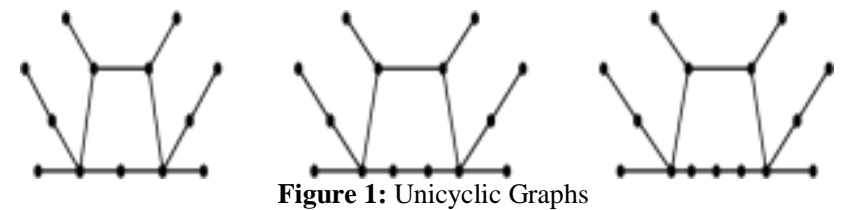
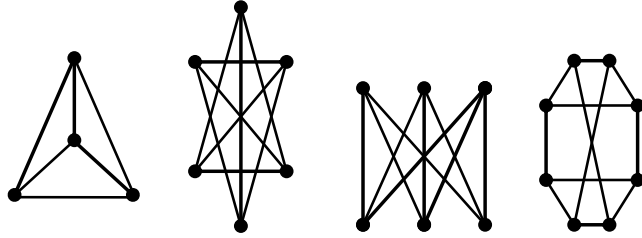

Figure 2: Cubic Graphs

\section{Main Results}

On studying the parameter, 'connected domination transition number' we are interested to characterize the class of graphs attaining the minimum or maximum bound. The case of studying the class of graphs attaining the minimum bound zero is equivalent to characterizing the class of graphs for which $\gamma=\gamma_{c}$. Many authors have attempted to study about this problem and characterized only some particular class of graphs having $\gamma=\gamma_{c}$. The following theorem characterizes such types of graphs.

\section{Theorem 4.1}

For any connected graph $G, \gamma(G)=\gamma_{c}(G)$ if and only if the following two conditions are satisfied:

i. $G$ has a spanning tree $T$, whose every internal vertex is a support.

ii. There exists a $\gamma$-set of $G$ constituted by the internal vertices of $T$.

\section{Proof}

Assume that $\gamma(G)=\gamma_{c}(G)$.

By theorem 3.3, for the connected domination number $\gamma_{c}(G)$ there corresponds a spanning tree $T$ of $G$ with (maximum) number of pendant vertices $\varepsilon_{T}(G)$ such that $\gamma_{c}(G)=n-\varepsilon_{T}(G)$. Using the relation (1), we have $\gamma(G)=n-\varepsilon_{T}(G)$. Hence, there corresponds a $\gamma$-set of cardinality $n-\varepsilon_{T}(G)$. We observe that the number $n-\varepsilon_{T}(G)$ is equal to the number of internal vertices of the spanning tree $T$. Let $D$ be the set of internal vertices of the spanning tree $T$. Obviously, the set of all internal vertices of any spanning tree is a dominating set and so $D$ is also a dominating set. Clearly the vertices of $D$ are connected. Hence the $\gamma$-set $D$ turns out to be a $\gamma_{c}$-set also satisfying the requirement (ii).

It remains to show that every internal vertex of $T$ is a support Otherwise, let $u$ be an internal vertex of $T$ that is not a support. Since the induced subgraph $\langle D\rangle$ is connected, $N(u) \cap D \neq \varphi$. It follows that, the set $D^{\prime}=D-\{u\}$ is also a dominating set whose cardinality is less than $\gamma$, which is a contradiction to the fact that $D$ is a minimal dominating set. Therefore, every internal vertex of the spanning tree $T$ is a support.
Conversely, assume that $G$ is a connected graph satisfying the requirements of the theorem.

Let $T$ be a spanning tree of $G$ whose every internal vertex of $T$ is a support (condition i). Let $D$ be a $\gamma$-set of $G$ formed by the internal vertices of $T$ (condition ii). Clearly, the induced subgraph $\langle D\rangle$ is connected. Hence $\langle D\rangle$ is also a $\gamma_{c}$-set . Therefore, we get $\gamma=\gamma_{c}$.

The above theorem is a more generalization of the characterization of graphs whose domination and connected domination numbers are equal. One can understand that this theorem is proved for any graph in general. And theorem 3.4, by Arumugam and Joseph [5], on class of trees follows as a corollary to the above theorem.

\section{Theorem 4.2}

For any connected graph $G, \gamma(G)=\gamma_{c}(G)$ if and only if $\mathrm{G}$ has some spanning tree $T_{G}$ with $n-\gamma(G)$ number of pendant vertices.

\section{Proof}

Let us assume that $\gamma(G)=\gamma_{c}(G)$

By theorem 3.3, we understand that corresponding to the connected domination number $\gamma_{c}(G)$, there exists some spanning tree $T$ of $G$ such that, $\gamma_{c}(G)=n-\varepsilon_{T}(G)$, where $\varepsilon_{T}(G)$ is the maximum number of vertices of any spanning tree of $G$. Since $\gamma(G)=\gamma_{c}(G)$, we see that the number of pendant vertices of the spanning tree $T$ is $n-\gamma(G)$.

Conversely, let $G$ be a connected graph having a spanning tree $T$ with $n-\gamma(G)$ pendant vertices. This implies that the number of internal vertices of the spanning tree $T$ is $\gamma(G)$ which are connected and hence these internal vertices form a $\gamma_{c}$-set also. Thus $\gamma(G)=\gamma_{c}(G)$
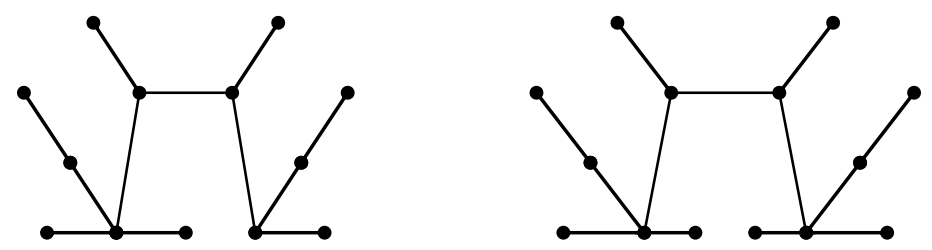

Figure 3: Spanning trees of unicyclic graphs
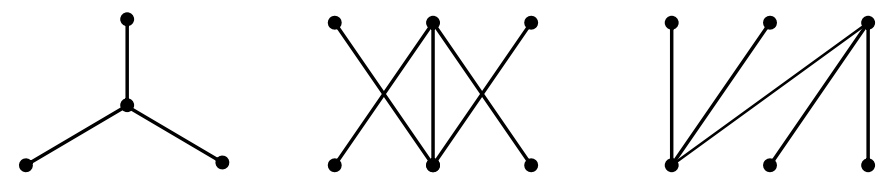

Figure 4: Spanning trees of cubic graphs

Examples of some graphs for which $\gamma(G)=\gamma_{c}(G)$

Though there are several graphs having $\gamma(G)=\gamma_{c}(G)$ to be brief enough, we consider the class of graphs considered by S.Arumugam in theorems 3.5 and 3.6 which are respectively unicyclic and cubic graphs.

The spanning trees of the graphs shown in figures 3 and 4 satisfy the conditions specified in theorems 4.1 and 4.2.

The following upper bound relates the domination number $\gamma$ with the connected domination transition number $\tau_{c}$.

\section{Theorem 4.3}

For any connected graph $G, \tau_{c}(G) \leq n-\left(\varepsilon_{T}(G)+1\right)$, where $\varepsilon_{T}(G)$ denotes the maximum number of pendant vertices (edges) in any spanning tree of $G$. 


\section{Proof}

From the definition of the parameter $\tau_{c}(G)$, one can understand that the above result is a direct consequence of theorem 3.3 and the fact that $\gamma(G) \geq 1$.

\section{Theorem 4.4}

For any connected graph $G, 0 \leq \tau_{c}(G) \leq 2(\gamma(G)-1)$. As noted earlier the lower bound is attained if and only if $\gamma(G)=\gamma_{c}(G)$. The upper bound is also sharp and is attained if and only if $d(u, v) \equiv 0(\bmod 3)$, for any two vertices $u$, v in any $\gamma-\operatorname{set} D$ of $G$.

\section{Proof}

We have already discussed the proof concerning the lower bound of the theorem. Let us proceed to discuss the proof concerning the upper bound and its related part.

Let $G$ be a connected graph with domination number $\gamma(G)$ and let $D$ be a $\gamma$-set of $G$.

If the induced subgraph $\langle D\rangle$ by itself is connected, then $\tau_{c}(G)=0$. This gives the lower bound of the inequality.

If the induced subgraph $\langle D\rangle$ is not connected then

(i) There exists at least one pair of vertices say $u, v \in D$ each of them belonging to different components of $\langle D\rangle$ such that $d(u, v) \leq 3 \quad($ see theorem 3.1$)$

(ii) It has at most $\gamma$ components in which case $D$ is independent. Let us form a subgraph $H$ of $G$ starting from the induced subgraph $\langle D\rangle$. Since $\langle D\rangle$ is disconnected, $H$ is also disconnected. By observation (i) any component of $H$ can be connected to some other component of $H$ by a path of length at most three. This path includes maximum two intermediate vertices between the components. Include this path in $H$. As long as $H$ is disconnected, inclusions of such paths are possible by observation (i).

By observation (ii) there are at most $\gamma$ components which can be minimally connected by the inclusion of at most $\gamma-1$ such paths (property of tree).

We see that the resulting subgraph $H$ is connected and the number of vertices included in $H$ in order to make it connected is at most $2(\gamma-1)$. In view of the remark 2.2 we obtain $\tau_{c}(G) \leq 2(\gamma(G)-1)$. We see that the path graphs $P_{3 k}, k=1,2,3, \ldots$ attain the upper bound and hence the bound is sharp. Let us continue the discussion about the attainment of the upper bound.

Let $D$ be any $\gamma$-set of $G$ such that $d(u, v) \equiv 0(\bmod 3)$, for any two vertices $u, v \in D$.

In this case, (a) the set $D$ is independent and (b) for every vertex $u \in D$, there exists at least one vertex $v \in D$ such that $d(u, v)=3$; otherwise, $D$ cannot be a dominating set. Hence any shortest $u-v$ path has exactly two intermediate vertices. The remaining part of the proof is easily follows from the above discussion.

Conversely, assume that $\tau_{c}(G)=2(\gamma(G)-1)$.

Let $D$ be a $\gamma$-set of $G$.

If the induced subgraph $\langle D\rangle$ is connected, then $\tau_{c}(G)=0$ which implies that $\gamma(G)=1$ and there is nothing to prove.

Suppose that the induced subgraph $\langle D\rangle$ is disconnected.

To prove: $d(u, v) \equiv 0(\bmod 3)$ for any two vertices $u, v \in D$.

On the contrary, assume that $d(u, v) \neq 0(\bmod 3)$, for some pair of vertices $u, v \in D$.

In the light of observation (i) above we see that $d(u, v) \leq 2$. Without loss of generality, let us assume that $d(u, v)=2$. Then the two components possessing the vertices $u, v$ can be connected by a path of length 2 which has only one intermediate vertex. In the light of observation (ii) above, the number of components in $\langle D\rangle$ yet to be connected is $\gamma-1$. From previous arguments all these components can be minimally connected by $(\gamma-2)$ path pieces each of which include at most two intermediate vertices. Hence the total number of intermediate vertices involved in the path pieces that connect the vertices of $D$ are $2(\gamma-2)+1=2 \gamma-3$. In view of the remark $2.2(2)$, we have $\tau_{c}(G)=2 \gamma-3$ which is less than $2(\gamma-1)$ a contradiction to the assumption. Hence the result follows.

Corollary 4.5 (Comparison with Independence number) $\tau_{c}(G) \leq 2(\beta(G)-1)$

\section{Proof}

We know that every maximal independent set is a minimal dominating set and hence $\gamma(G) \leq \beta(G)$, where $\beta(G)$ denotes the independence number of the graph $G$. Using this in the above inequality we get, $\tau_{c}(G) \leq 2(\beta(G)-1)$.

The same holds for the lower independence number $i(G)$ (which is also called as independent domination number) and hence we get another similar corollary.

Corollary 4.6(Comparison with Independent domination number) $\tau_{c}(G) \leq 2(i(G)-1)$

Results on Tree graphs

Though we have so far derived results on the bounds of the parameter, connected domination transition number of a graph $\tau_{c}(G)$, using its spanning trees, we have not discussed yet the bounds of the class of graphs called trees.

\section{Theorem 4.7}

If $T$ is a tree with $l$ pendant vertices, then $\tau_{c}(T) \leq \frac{2(n-l-1)}{3}$. The bound is reached if and only if $\mathrm{R}$ includes the tree $T$ where $\mathrm{R}$ is the family of all trees in which for any two distinct pendant vertices $u$ and $v, d(u, v) \equiv 2(\bmod 3)$.

\section{Proof}

Since the connected domination number for trees remains to be constant as $\gamma_{c}=n-l$ by theorem 3.2, the case of attaining the upper bound for the connected domination transition number of trees $\tau_{c}(T)$ wholly lies on the case of tree attaining the lower bound of the value $\gamma(T)$. The remaining part of the theorem follows easily from theorem 3.7.

\section{Theorem 4.8}

For any integer $k \geq 0$, there exists a graph such that $\tau_{c}(G)=k$.

\section{Proof}

For $k=0$, let $G$ be a corona graph $P_{k} \circ K_{1}$ then $\gamma(G)=\gamma_{c}(G)$ and $\tau_{c}(G)=0$.

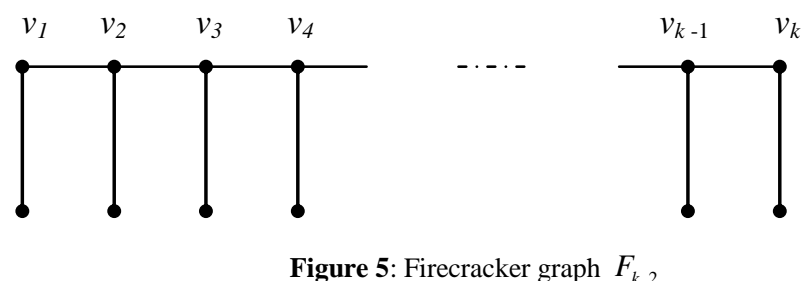

For $k=1$, let $G^{\prime}$ be the path graph $P_{5}$. 
For $k>1$, , let $G^{\prime \prime}$ be the graph obtained from $G$ by subdividing the pendant edges.
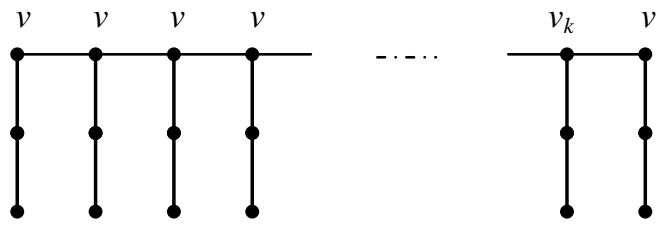

Figure 6: Firecracker graph $F_{k, 3}$

It is an easy exercise to anyone to verify that $\tau_{c}\left(G^{\prime \prime}\right)=k, k>1$.

Thus the graphs $G, G^{\prime}, G^{\prime \prime}$ render $\tau_{c}(G)=k$, for all nonnegative integers $k$.

Nordhaus-Gauddam Type Results

We wish to discuss a NG (Nordhaus-Gauddam) result [10] for the parameter $\tau_{c}(G)$. The NG results we have recalled in section 4 of this chapter, for both the domination parameters $\gamma(G)$ and $\gamma_{c}(G)$ are of upper bound types. In view of the remark 2.3 , in order to derive one such upper bound we require a NG result on the lower bound of the parameter $\gamma(G)$.

In order to use deduce some NG results we require a lower bound result of same type for the domination parameter as in the next theorem.

\section{Theorem 4.9}

If $G$ is a coconnected graph, then $\gamma+\bar{\gamma} \geq 4$ and the bound is sharp.

\section{Proof}

Let $G$ be a coconnected graph. This implies both graphs $G$ and $\bar{G}$ are connected and $n \geq 4$. This implies none of the graphs $G$ and $\bar{G}$ has a vertex of full degree; otherwise, if $v \in G$ is a vertex of full degree i.e. $d(v)=n-1$, then it is adjacent to every vertex of $G$ by which it would be isolated from the remaining vertices in the complement graph $\bar{G}$, a contradiction to the assumption that both $G$ and $\bar{G}$ are connected. Similar argument holds for the complement graph $\bar{G}$ also.

Thus $d(v)<n-1$, for any vertex $v \in G, \bar{G}$.

Hence $\gamma(G) \geq 2$ and $\gamma(\bar{G}) \geq 2$.

i.e. $\gamma, \bar{\gamma} \geq 2$ and hence $\gamma+\bar{\gamma} \geq 4$.

When $G=P_{4}$, we see that $\gamma+\bar{\gamma}=4$. Hence the bound is sharp.

Theorem 4.10

If $G$ is a coconnected graph, then $\tau_{c}+\bar{\tau}_{c} \leq(n+1)(n-4)$, where $\tau_{c}=\tau_{c}(G)$ and $\bar{\tau}_{c}=\tau_{c}(\bar{G})$ and the bound is sharp.

\section{Proof}

Since $G$ is a coconnected graph, both graphs $G$ and $\bar{G}$ are also connected and $n \geq 4$. By definition we have, $\tau_{c}(G)=\gamma_{c}(G)-\gamma(G)$ and $\tau_{c}(\bar{G})=\gamma_{c}(\bar{G})-\gamma(\bar{G})$

i.e. $\tau_{c}+\bar{\tau}_{c}=\tau_{c}(G)+\tau_{c}(\bar{G})$

$=\gamma_{c}(G)-\gamma(G)+\gamma_{c}(\bar{G})-\gamma(\bar{G})$

$=\gamma_{c}(G)+\gamma_{c}(\bar{G})-(\gamma(G)+\gamma(\bar{G}))$

$\leq n(n-3)-4=n^{2}-3 n-4=(n+1)(n-4)$, using theorems 3.9 and 4.9 .

Again the path $P_{4}$ attains the bound. Hence this bound is sharp.

\section{Theorem 4.11}

If any graph $G$ is coconnected, then $\tau_{c}+\bar{\tau}_{c} \leq n-3$ and the bound is sharp.

Proof

As in the previous theorem we have,

$\tau_{c}+\bar{\tau}_{c}=\gamma_{c}(G)+\gamma_{c}(\bar{G})-(\gamma(G)+\gamma(\bar{G}))$

Using the theorems 3.10 and 4.9 we get, $\tau_{c}+\bar{\tau}_{c} \leq n-3$.

When $G=C_{5}$, the upper bound in above bound is reached.

\section{Conclusion}

In this research article we have focused primarily on establishing a stable and reliable facility location from an existing one by means of constructing connected dominating sets in the networks. To this end, researchers have defined a new domination related parameter viz., connected domination transition number of a graph and studied its properties. We add flavor to this parameter by exploring its bounds and characteristics for various types of graphs. We also have attempted on Nordhaus-Gauddam type results for the variant.

\section{References}

[1] C. Berge, 1962: Theory of Graphs and its Applications. Methuen, London.

[2] O. Ore, 1962: Theory of Graphs. Amer. Math. Soc. Colloq. Publ., 38, Providence.

[3] E. Sampathkumar, and H.B. Walikar, 1979: The connected domination number of a graphs. J. Math. Phys. Sci., 13(6), 607-613.

[4] S.T.Hedetniemi and R.C.Laskar, 1984: Connected domination in Graphs. Graph Theory and Combinatorics, B.Bollobas, Ed., Academic press, London, 209-218.

[5] S. Arumugam, and P. Joseph, 1999: On graphs with equal domination and connected domination numbers. Discrete Math., 206, 4549.

[6] Kaspar S, B. Gayathri, M.P. Kulandaivel, N. Shobanadevi J.Nieminen, 2017: Towards connected domination in graphs. Int. J. Pure and Applied Math., 117(14), 53-62.

[7] R. Laskar and K. Peters, 1985: Vertex and edge domination parameters in graphs. Congr. Numer., 48, 291-305.

[8] F. Harary and T.W. Haynes, 1996: Nordhaus-Gaddum inequalities for domination in graphs. Discrete Math., 155, 99-105.

[9] M. Lemanska, 2004: Lower bound on the domination number of a tree. Discuss. Math. Graph Theory, 24, 165-169.

[10] E.A. Nordhaus and J.W Gaddum, 1956: On complementary graphs. Amer. Math. Monthly, 63, 175-177.

[11] F. Jaeger and C. Payan, 1972: Relations du type Nordhaus-Gaddum pour le nombre d'absorption d'un graphe simple. C.R. Math. Acad. Sci. Paris, A 274, 728-730.

[12] E.J. Cockayne, and S.T. Hedetniemi, 1977: Towards the theory of domination in graphs. Networks, 7, 247-261. 